\title{
A POSSIBLE EVOLUTIONARY SCENARIO FOR NGC 7217
}

\author{
E. ATHANASSOULA, A. BOSMA AND B. GUIVARCH \\ Observatoire de Marseille \\ 2 Place Le Verrier, 13248 Marseille Cedex 4, FRANCE \\ AND \\ L. VERDES-MONTENEGRO \\ Instituto di Astrofisica di Andalucia \\ Apdo. Correos 3004, E-18080 Granada, SPAIN
}

NGC 7217 is an ordinary spiral galaxy with three rings whose size ratios are such that they can be associated with resonances, as for barred spirals. From 21-cm HI line data and BVRI CCD-images of this galaxy we find (cf. Verdes-Montenegro et al. 1995) : 1) a nuclear ring strong in $\mathrm{H} \alpha, 2$ ) an inner ring seen clearly in a B - I colour map, and 3) an outer ring, with blue colours and strong HI-emission. After deprojection the disk has a mean ellipticity of $0.04 \pm 0.01$, while the position angle of the deprojected galaxy changes suddenly at 65 " radius, where the minor axis becomes major axis. Thus a very mild oval distortion could exist, with the outer ring perpendicular to the oval. Merrifield and Kuijken (1994) find from the stellar kinematics that about $20-30 \%$ of the stars are in retrograde orbits.

Did NGC 7217 have a bar which has since been destroyed ? This is possible : 1) From a simulation with sticky particles we find that rings can survive quite long after a bar has been turned off. 2) Orbit calculations show that a large fraction of the ergodic orbits present before a bar is switched off become retrograde. Thus if the bar had a substantial fraction of ergodic orbits, the presence of the retrograde orbits follows from a pendulum analogy.

There are several ways to decay the bar : 1) N-body simulations show that by hitting a barred galaxy with a companion the bar can be destroyed, and in such cases a substantial fraction of the stars is moving in retrograde orbits, as in NGC 7217.2) A build up of a strong central mass concentration can scatter the $\mathrm{x}_{1}$-orbits, while temporarily increasing the fraction of ergodic orbits. 